\title{
Pyramid-based texture synthesis using local orientation and multidimensional histogram matching
}

\author{
Dimitri Van De Ville ${ }^{1,2}$, Matthieu Guerquin-Kern ${ }^{1}$, Michael Unser ${ }^{1}$ \\ ${ }^{1}$ École Polytechnique Fédérale de Lausanne (EPFL), Switzerland \\ ${ }^{2}$ University of Geneva, Switzerland
}

\begin{abstract}
One very influential method for texture synthesis is based on the steerable pyramid by alternately imposing marginal statistics on the image and the pyramid's subbands. In this work, we investigate two extensions to this framework. First, we exploit the steerability of the transform to obtain histograms of the subbands independent of the local orientation; i.e., we select the direction of maximal response as the reference orientation. Second, we explore the option of multidimensional histogram matching. The distribution of the responses to various orientations is expected to capture better the local geometric structure. Experimental results show how the proposed approach improves the performance of the original pyramid-based synthesis method.
\end{abstract}

Keywords: Multiresolution analysis, steerable filters, texture synthesis, multidimensional histogram matching

\section{INTRODUCTION}

Texture synthesis has attracted a lot of attention in the fields of computer graphics, vision, and image processing. In most of the methods that have been investigated over the years, statistical models play a central role. ${ }^{1}$ These models try to characterize the texture by a limited number of parameters. Initially, Markov random fields were applied to describe (and reproduce) statistical interactions within local neighborhood. ${ }^{2-4}$ Later on, mainly inspired by psychophysics, the field of texture classification successfully deployed multiresolution representations,${ }^{5,6}$ including the wavelet decomposition. ${ }^{7}$ The same concepts were then introduced for texture synthesis as well. ${ }^{8-10}$

A simple and elegant method for stochastic texture synthesis was proposed by Heeger and Bergen. ${ }^{11}$ The reference texture is characterized by its histograms in the image domain and each subband of steerable pyramid decomposition. Then, the new texture is synthesized from uniform white noise by alternately matching the histograms in the image domain and in the transformed domain. This method works well for stochastic textures and empirically converges after few iterations. While there is no explicit modeling of (in-band) correlation, the multiresolution structure is supposed to induce spatially correlated structure. In Fig. 1, we show an example of this method. Portilla and Simoncelli ${ }^{12}$ generalized this method by imposing joint statistics (e.g., in-band and cross-scale correlations), resulting in the generated textures that display much more geometric structure.

In this paper, we focus on stochastic methods to generate texture out of noise. We explore two extensions of the original pyramid-based approach by Heeger and Bergen, ${ }^{11}$ while maintaining the method's simplicity and its easiness of implementation.

- The most powerful feature of the steerable pyramid is its orientation shiftability; i.e., the response can be oriented in any direction by a linear transformation matrix. We want to exploit this property to decouple the histogram description from the local orientation. Here, we will maximize the response along the main orientation of the steerable pyramid.

- We also want to exploit information that is captured by correlation between the orientations. Instead of sequential 1-D histogram matching along all orientations, we propose multidimensional histogram matching.

After discussing the various elements of our framework in Section 2, we show and discuss experimental results in Section 3.

Further author information: (dimitri.vandeville, matthieu.guerquin-kern, michael.unser)@epfl.ch

Wavelets XIII, edited by Vivek K. Goyal, Manos Papadakis, Dimitri Van De Ville, Proc. of SPIE

Vol. 7446, 74460X - @ 2009 SPIE · CCC code: 0277-786X/09/\$18 - doi: 10.1117/12.826812 


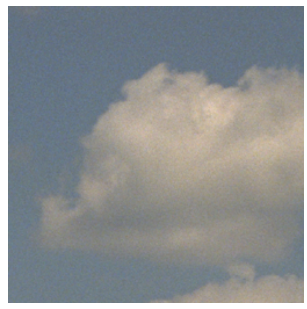

(a)

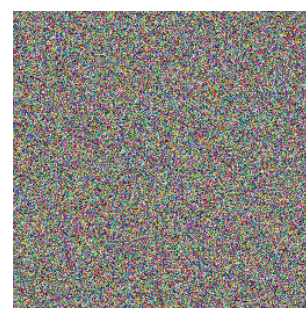

(b)

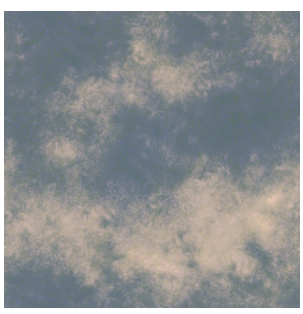

(c)

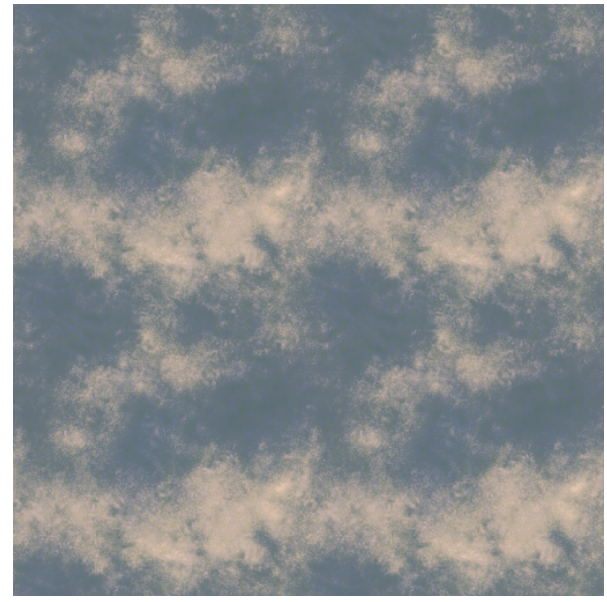

(d)

Figure 1. Example of stochastic texture synthesis using Heeger and Bergen's method. (a) Reference texture. (b) Uniform white noise initialisation. (c) Generated texture. (d) Seemless tiling due to periodic boundary conditions that were used for generating (c).

\section{OVERVIEW OF THE TEXTURE SYNTHESIS ALGORITHM}

\subsection{Steerable pyramid}

The steerable pyramid is a multiscale and multi-orientation linear transform. ${ }^{13,14}$ It constitutes a tight frame that has shift-invariant and rotation-invariant (but steerability) properties. For $K$ orientations, the redundancy factor is limited to $4 K / 3+1$. The equivalent basis functions of the steerable pyramid are localized directional derivatives (of order $K-1$ ). For instance, $K=2$ gives a classical gradient-type pyramid decomposition.

While we refer to the references ${ }^{13,14}$ for a detailed description of the steerable pyramid and its properties, we want to highlight the main feature for this work. Specifically, the core of the filterbank contains bandpass filters that are steered versions of a single generating filter; i.e., in polar-separable form, the Fourier expressions of the bandpass filters for $K$ orientations are

$$
B_{m}(\boldsymbol{\omega})=\left(-j \cos \left(\theta-\theta_{m}\right)\right)^{K-1} B(\omega), \quad m=1, \ldots, K,
$$

where $\theta=\tan ^{-1}\left(\omega_{2} / \omega_{1}\right), \omega=\|\boldsymbol{\omega}\|, \theta_{m}=(m-1) \pi / K$, and $B(\omega)$ is the radial profile. The filters $B_{m}(\boldsymbol{\omega})$ can be turned into any orientation $\tilde{\theta}$ by the steering relation

$$
B_{m, \tilde{\theta}}(\boldsymbol{\omega})=\sum_{k=1}^{K} h_{m, k}(\tilde{\theta}) B_{k}(\boldsymbol{\omega})
$$

where the steering kernels $h_{m, k}(\tilde{\theta})$ can be found by solving for the identities:

$$
\sum_{k=1}^{K} h_{m, k}(\tilde{\theta})\left(-j \cos \left(\theta-\theta_{m}\right)\right)^{K-1}=\left(-j \cos \left(\theta-\tilde{\theta}-\theta_{m}\right)\right)^{K-1}, \quad m=1, \ldots, K .
$$

We denote the coefficients of the steerable pyramid as $w_{m}^{(j)}[\mathbf{k}]$, where $j$ is the decomposition level, $m$ is the orientation, and $\mathbf{k}$ is the in-band position. We also write the vector $\mathbf{w}^{(j)}[\mathbf{k}]$ that contains the coefficients of all orientations at scale $j$ and position $\mathbf{k}$.

\subsection{Steering property}

Orientation shiftability is probably the most attractive property of the steerable pyramid. Given coefficients of the pyramid at various orientations for a fixed scale $j$ and position $\mathbf{k}$, the optimal angle can be found; i.e., we 


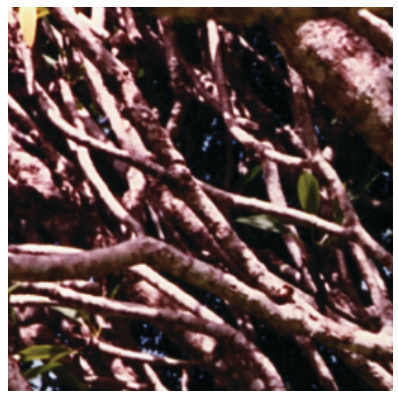

(a)

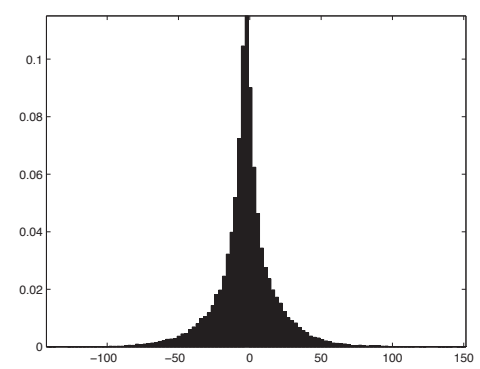

(b)

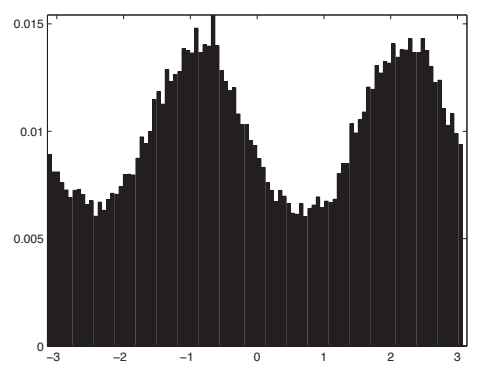

(d)

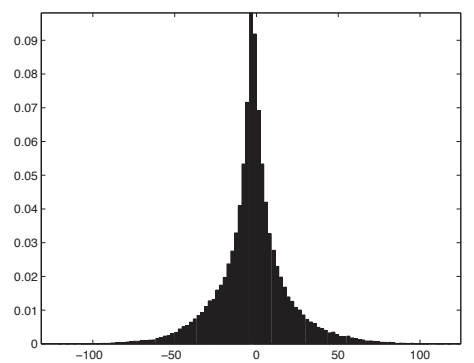

(c)

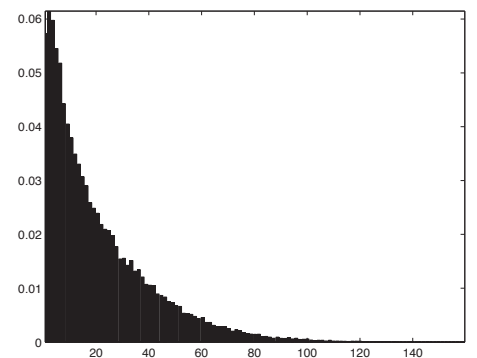

(e)

Figure 2. Example illustrating the principle of steered histogram. (b) and (c) show the marginal histograms for the coefficients of the first level of the steerable pyramid $(K=2)$ of image (a). (d) shows the histogram of the optimal orientation. The histogram of the coefficient after reorienting the pyramid is shown in (e).

look for the orientation angle $\theta_{\max }$ that maximizes the response in the first channel:

$$
\left.\theta_{\max }\right|_{j, \mathbf{k}}=\arg \max _{\tilde{\theta}} \sum_{n=1}^{K} h_{1, n}(\tilde{\theta}) w_{n}^{(j)}[\mathbf{k}] .
$$

The optimal angle can be found among the roots of a $(K-1)$-th degree polynomial. Each coefficient vector $\mathbf{w}^{(j)}[\mathbf{k}]$ can then be steered to $\tilde{\mathbf{w}}^{(j)}[\mathbf{k}]$ accordingly, before constructing the histograms. To illustrate this principle, in Fig. 2, we show an example for $K=2$ (gradient-style pyramid). For the test image in (a), the histograms of the coefficients $w_{1}^{(1)}[\mathbf{k}]$ and $w_{2}^{(1)}[\mathbf{k}]$ are shown in (b) and (c), respectively. Despite the apparent strong edges in some directions, no clear trend is visible is these histograms. In (d), we show the histogram of the angle of maximal response that clearly reveals the preferential orientations. In (e), we show the histogram of $\tilde{w}_{1}^{(1)}[\mathbf{k}]$, while in the case $K=2$ all coefficients $\tilde{w}_{2}^{(2)}[\mathbf{k}]$ become zero after steering (a well known property in differential geometry). The histograms (d) and (e) are able to disentangle better the information of the pyramid's coefficients.

\subsection{Multidimensional histogram matching}

The purpose of multidimensional histogram matching (along the orientations) is to better reproduce the local geometric structure of the reference texture. Let us first start by recalling the traditional histogram, which can be interpreted (after normalization) as an estimator for the probability density function (pdf) of a coefficient's intensity; i.e., we denote the pdf and cumulative distribution function (cdf) as

$$
f_{j, m}(W) d W=P\left(W<w_{m}^{(j)}[\mathbf{k}]<W+d W\right), \quad F_{j, m}(W)=P\left(w_{m}^{(j)}[\mathbf{k}] \leq W\right) .
$$

To match the histograms from a source and a reference, characterized by the cdfs $F_{j, m}^{S}$ and $F_{j, m}^{R}$, respectively, we find the classical mapping function ${ }^{15}$

$$
W_{\text {new }}=\left(F_{j, m}^{R}\right)^{-1} F_{j, m}^{S}\left(W_{S}\right) .
$$


Inverting $F_{j, m}^{R}$ is only unique when $F_{j, m}^{R}$ is strictly monotonous. A more general form consists of looking for the lowest value of $W$ for which $F_{j, m}^{R}(W) \geq F_{j, m}^{S}\left(W_{S}\right)$, which translates in the following mapping function:

$$
W_{\text {new }}=\arg \min _{W}\left(F_{j, m}^{R}(W) \geq F_{j, m}^{S}\left(W_{S}\right)\right) .
$$

Histogram matching has been extended to the multidimensional case,${ }^{16,17}$ mostly for the purpose of the color image processing. ${ }^{18}$ The pdf and cdf can be easily extended to the multidimensional case:

$$
\begin{aligned}
f_{j}(\mathbf{W}) d W_{1} \ldots d W_{K} & =P\left(W_{1}<w_{1}^{(j)}[\mathbf{k}]<W_{1}+d W_{1}, \ldots, W_{K}<w_{K}^{(j)}[\mathbf{k}]<W_{K}+d W_{K}\right), \\
F_{j}(\mathbf{W}) & =P\left(w_{1}^{(j)}[\mathbf{k}] \leq W_{1}, \ldots, w_{K}^{(j)}[\mathbf{k}] \leq W_{K}\right) .
\end{aligned}
$$

Further on, the joint cdf can be rewritten as

$$
F_{j}(\mathbf{W})=\prod_{n=1}^{K} \underbrace{P\left(w_{n}^{(j)}[\mathbf{k}] \leq W_{n} \mid \bigwedge_{n^{\prime}=1}^{n-1} w_{n^{\prime}}^{(j)}[\mathbf{k}]=W_{n^{\prime}}\right)}_{\tilde{F}_{j, n}\left(W_{n} ; W_{1}, \ldots, W_{n-1}\right)},
$$

from which one can derive that multidimensional histogram matching can be performed by $K$ sequential 1-D histogram matchings:

$$
\begin{aligned}
W_{\text {new }, 1} & =\arg \min _{W}\left(F_{j, 1}^{R}(W) \geq F_{j, 1}^{S}\left(W_{S, 1}\right)\right) \\
W_{\text {new }, 2} & =\arg \min _{W}\left(\tilde{F}_{j, 2}^{R}\left(W ; W_{\text {new }, 1}\right) \geq \tilde{F}_{j, 2}^{S}\left(W_{S, 2} ; W_{S, 1}\right)\right), \\
& \vdots \\
W_{\text {new }, K} & =\arg \min _{W}\left(\tilde{F}_{j, K}^{R}\left(W ; W_{\text {new }, 1}, \ldots, W_{\text {new }, K-1}\right) \geq \tilde{F}_{j, K}^{S}\left(W_{S, K} ; W_{S, 1}, \ldots, W_{S, K-1}\right)\right) .
\end{aligned}
$$

We illustrate the matching procedure for $K=2$ in Fig. 3. The 2-D histograms of the source and the reference texture are shown in the top row. The first 1-D histogram matching operates on the marginal statistics of $W_{1}$. The second matching uses the 1-D histograms of $W_{2}$ that are conditional to $W_{S, 1}$ and $W_{\text {new,1 }}$, respectively.

\subsection{Texture matching algorithm}

We now have all elements to put together the texture matching algorithm. The pseudo-code below shows how subbands are steered according to the direction of maximal response. Next, multidimensional histogram matching is performed and the steering is "undone" according to the transfer function that matches the angles' distribution of the reference texture.

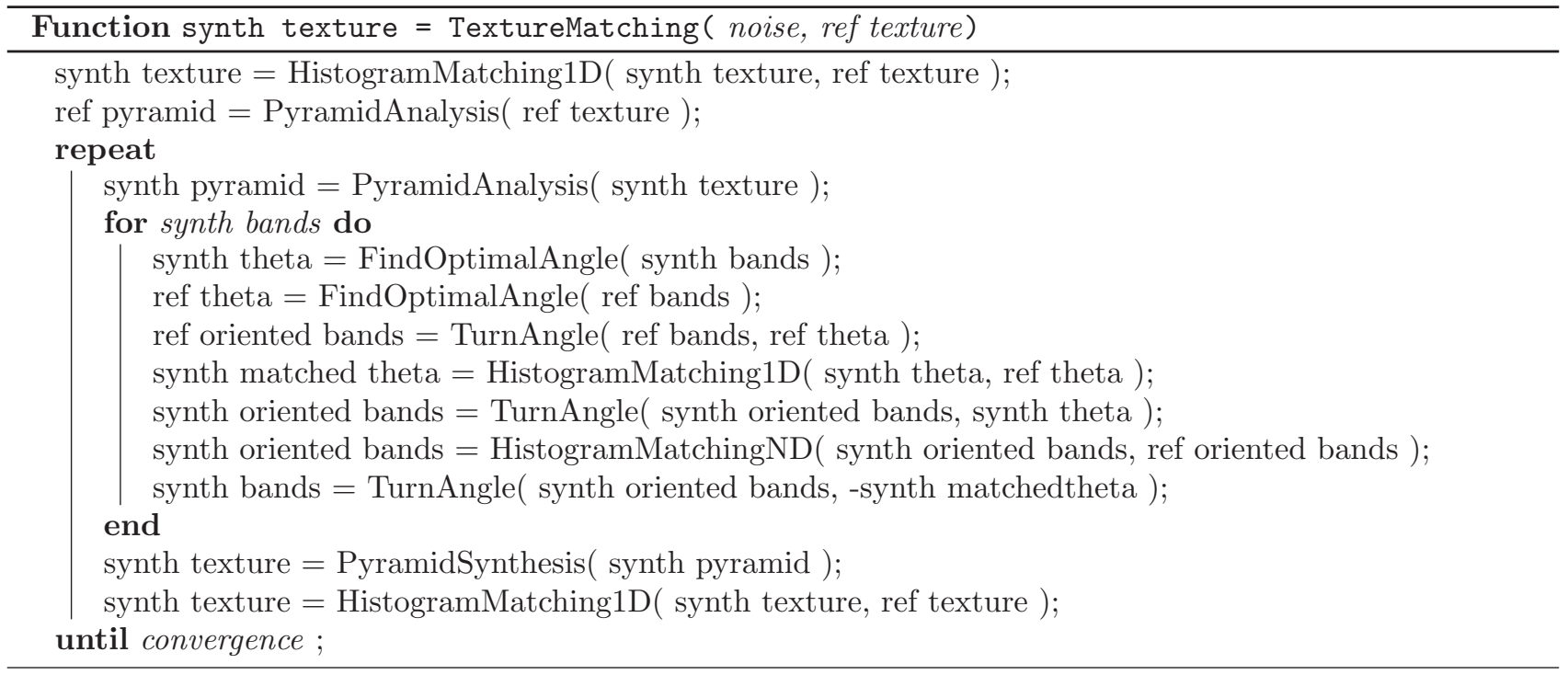



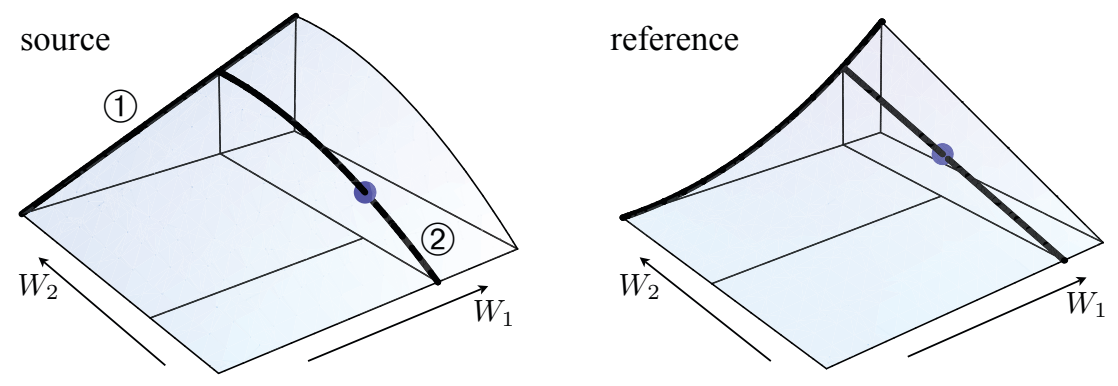

(1)
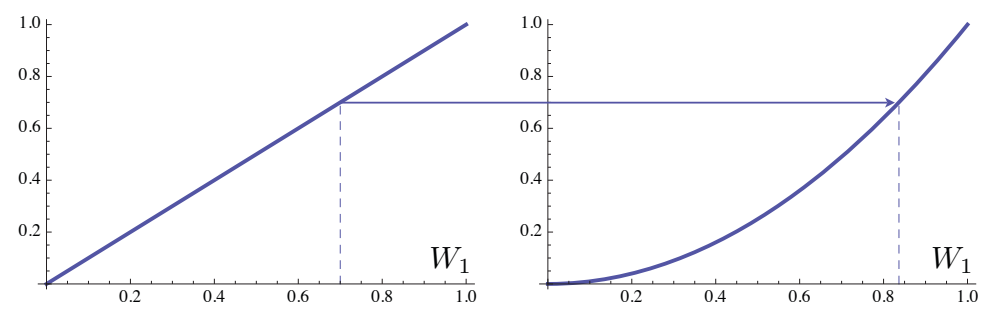

(2)
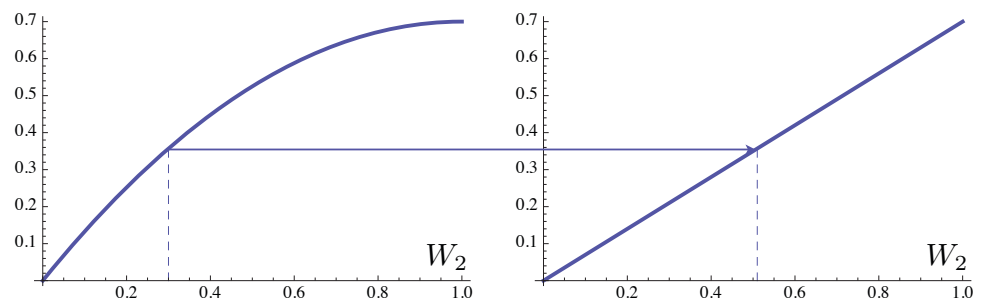

Figure 3. The multidimensional histogram matching is illustrated in 2-D.

\section{RESULTS AND DISCUSSION}

For the experimental results, we used texture images from the VisTex database*. To apply our algorithm to color textures, we first decorrelated the RGB components of the reference texture using a principal component analysis. The algorithm was then applied to each channel independently, while keeping the same noise initialisation. Finally, the channels of the generated texture were put together using the inverse of the color decorrelating matrix. When comparing the different methods, we always used the same noise initialisation.

As we observe from the results in Fig. 4, the steering mechanism improves the "edginess" of the generated texture; i.e., both (c) and (d) show a better edge contrast similar to the reference texture. The storage needed for the multidimensional histograms, at fixed quantization quality (number of bins) in each dimension, increases exponentially with the number of orientations. For example, 100 bins and 4 orientations already require $100 \mathrm{M}$ entries. We empirically found good results for 50 bins in each dimension.

From the results in Fig. 5, we observe that the steered multidimensional solution in (d) has the best color contrast and also the "grainy" appearance of the reference texture is reproduced. In Fig. 6, we show the effect of changing the number of orientations $(K=2,3,4)$. More orientations allows to better characterize the directionality of the texture at larger spatial extent.

Finally, the example in Fig. 7 shows a failure. Nevertheless, the steering and multidimensional histogram matching show more coherent structures.

Future research could investigate in more detail the influence of the various parameters, such as the number of orientations and the number of bins. A possible solution to circumvent the quantization problem related to multidimensional histograms could be Parzen window estimation or parametric models. Another interesting

\footnotetext{
${ }^{*}$ Available at http://vismod.media.mit.edu/vismod/imagery/VisionTexture/vistex.html.
} 


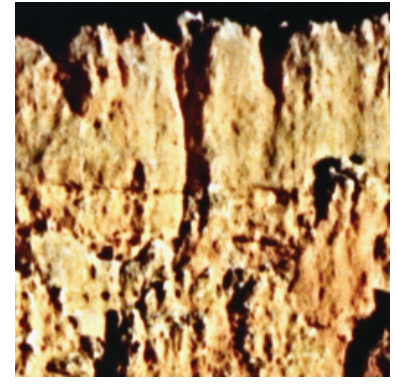

(a)

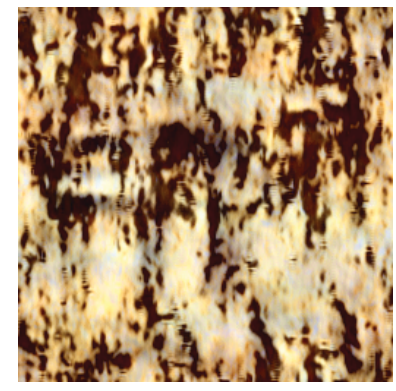

(b)

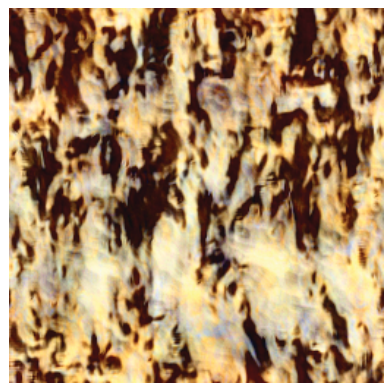

(c)

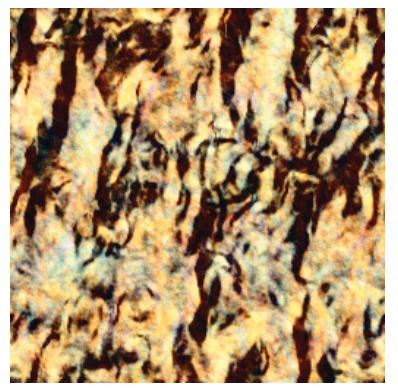

(d)

Figure 4. Results for steerable pyramid with $K=4$ orientations and 6 decomposition levels. The image sizes are $256 \times 256$. 40 iterations for all methods. (a) Original texture (Stone.0001). (b) No steering, no multidimensional histogram matching. (c) Steering, no multidimensional histogram matching. (d) Steering, multidimensional histogram matching.

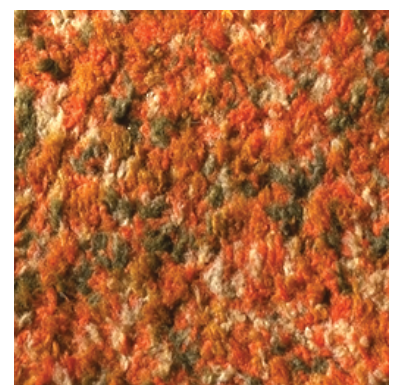

(a)

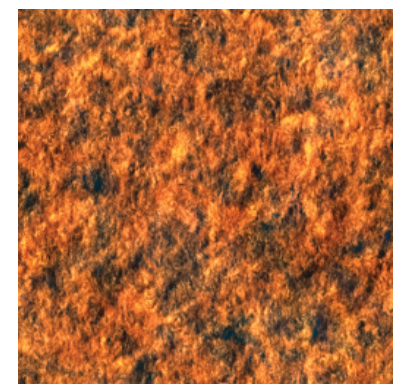

(b)

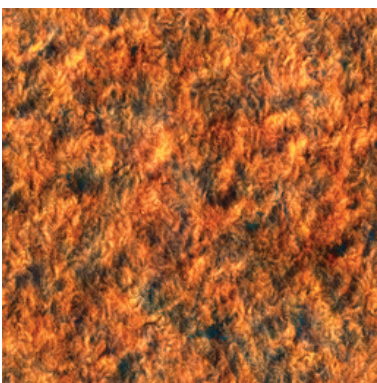

(c)

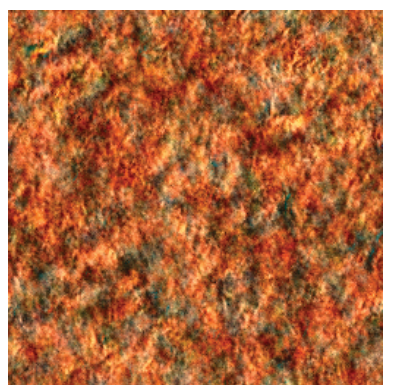

(d)

Figure 5. Results for steerable pyramid with $K=4$ orientations and 6 decomposition levels. The image sizes are $256 \times 256$. 20 iterations for all methods. (a) Original fabric texture (Fabric.0016). (b) No steering, no multidimensional histogram matching. (c) Steering, no multidimensional histogram matching. (d) Steering, multidimensional histogram matching.

topic is the convergence - empirically, we observed convergence of the algorithms after 20-40 iterations. Finally, it should be noted that recently another class of texture synthesis methods received a lot of attention. They take patches from the original reference texture and use them as building blocks for the generated texture. ${ }^{19,20}$ These methods work very well for (near-)regular textures since they maintain the local structure, but they do not contribute to a better understanding of the parameters that characterize the texture. An outstanding question is whether and how both methodologies - stochastic texture generation and resampling of the reference texture - can be combined advantageously.

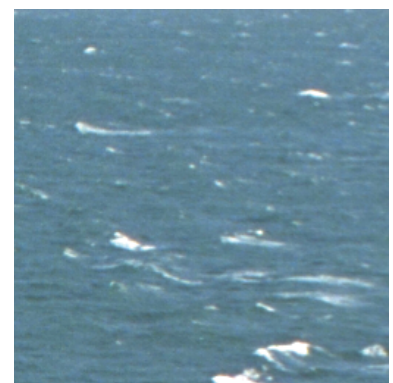

(a)

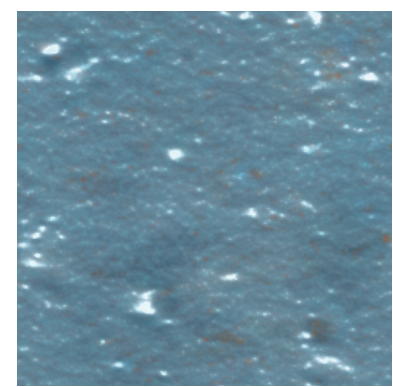

(b)

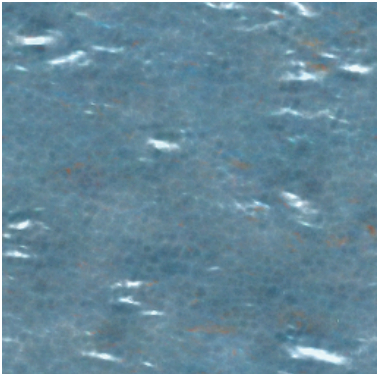

(c)

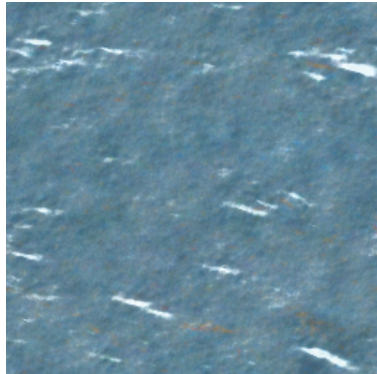

(d)

Figure 6. Effect of changing the number of orientations. (a) Original water texture (Water.0002). (b) $K=2$. (c) $K=3$. (d) $K=4$. 


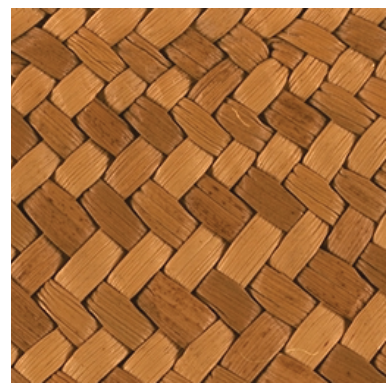

(a)

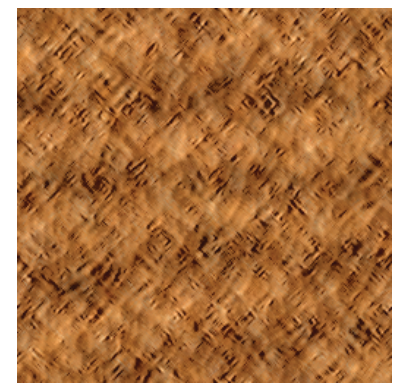

(b)

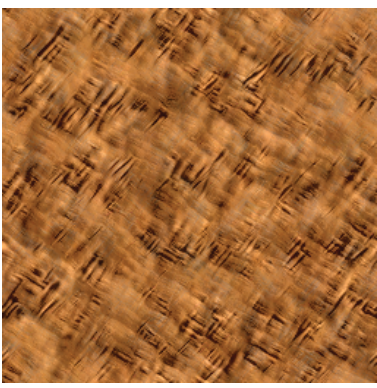

(c)

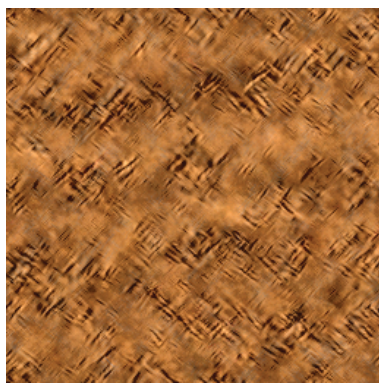

(d)

Figure 7. Example of failure. It is interesting, however, to observe the partial coherent structures that appear using the steering extension. Results for steerable pyramid with $K=4$ orientations and 6 decomposition levels. The image sizes are $256 \times 256$. 40 iterations for all methods. (a) Original fabric texture (Fabric.0000). (b) No steering, no multidimensional histogram matching. (c) Steering, no multidimensional histogram matching. (d) Steering, multidimensional histogram matching.

\section{ACKNOWLEDGMENTS}

This work was supported in part by the Swiss National Science Foundation under grants PP00P2-123438 and 200020-109415, and in part by the Center for Biomedical Imaging (CIBM) of the Geneva - Lausanne Universities and the EPFL, the foundations Leenaards and Louis-Jeantet.

\section{REFERENCES}

1. H. Iversen and T. Lonnestad, "An evaluation of stochastic models for analysis and synthesis of gray-scale texture," Pattern Recognition Letters 15, pp. 575-585, 1994.

2. R. Yokoyama and R. M. Haralick, "Texture pattern image generation by regular Markov chain," Pattern Recognition 11, pp. 225-233, 1979.

3. M. Hassner and J. Sklansky, "The use of Markov random fields as models of texture," Comp. Graphics Image Proc 12, pp. 357-370, 1980.

4. G. Cross and A. Jain, "Markov random field texture models," IEEE Transactions on Pattern Analysis and Machine Learning 5, pp. 25-39, 1983.

5. J. R. Bergen and E. H. Adelson, "Visual texture segmentation based on energy measures," J. Opt. Soc. Am. A 3, p. 99, 1986.

6. M. Turner, "Texture discrimitation by Gabor functions," Biol. Cybern. 55, pp. 71-82, 1986.

7. M. Unser, "Texture classification and segmentation using wavelet frames," IEEE Transactions on Image Processing 4, pp. 1549-1560, November 1995.

8. D. Cano and T. H. Minh, "Texture synthesis using hierarchical linear transforms," Signal Processing 15, pp. 131-148, 1988.

9. M. Porat and Y. Y. Zeevi, "Localized texture processing in vision: Analysis and synthesis in Gaborian space," IEEE Trans. Biomedical Eng. 36, pp. 115-129, 1989.

10. K. Popat and R. W. Picard, "Novel cluster-based probability model for texture synthesis," in Proc. SPIE Vis Comm., 1993.

11. D. J. Heeger and J. R. Bergen, "Pyramid-based texture analysis/synthesis," Computer Graphics Proceedings , pp. 229-238, 1995.

12. J. Portilla and E. P. Simoncelli, "A parametric texture model based on joint statistics of complex wavelet coefficients," International Journal of Computer Vision 40(1), pp. 49-71, 2000.

13. E. P. Simoncelli, W. T. Freeman, and E. H. Adelson, "Shiftable multi-scale transforms," IEEE Transactions on Information Theory 38, pp. 587-607, Mar. 1992.

14. E. Simoncelli and W. Freeman, "The steerable pyramid: a flexible architecture for multi-scale derivative computation," in International Conference on Image Processing, 3, pp. 444-447, 23-26 October 1995. 
15. E. S. Pearson, "The probability integral transformation for testing goodness of fit and combining independent tests of significance," Biometrika 30, pp. 134-148, 1938.

16. J. M. Soha and A. A. Schwartz, "Multidimensional histogram normalization contrast enhancement," in Proc. 5th Canadian Symp. Remote Sensing, pp. 86-93, 1978.

17. C. Genest and L.-P. Rivest, "On the multivariate probability integral transformation," Statistics and Probability Letters 53(4), pp. 391-399, 2001.

18. L. Neumann and A. Neumann, "Color style transfer techniques using hue, lightness and saturation histogram matching," in Computational Aesthetics in Graphics, Visualization and Imaging 2005, L. Neumann, M. Sbert, B. Gooch, and W. Purgathofer, eds., pp. 111-122, 52005.

19. A. Efros and T. Leung, "Texture synthesis by non-parametric sampling," in International Conference on Computer Vision, 2, pp. 1033-1038, 1999.

20. A. A. Efros and W. T. Freeman, "Image quilting for texture synthesis and transfer," in Proceedings of SIGGRAPH' '01, 2001. 